R. J. Cohen and W. T. Sullivan, III, eds.

\title{
Radio Astronomy and Recent Telecommunications Trends
}

\author{
Tomas E. Gergely \\ National Science Foundation, Arlington, VA 22230, USA
}

\begin{abstract}
Radio astronomy was born in the 1930s and matured in the 1950s. The telecommunications environment of those decades was dominated by monopolies (often state monopolies) and a strong regulatory environment. Radio communications were analogue in nature and mostly confined to frequencies below a few hundred megahertz. The worldwide telecommunications environment began to change in the 1960 s and has undergone a revolution during the last three decades. Impulse for this revolution, which has not yet ended, was provided by political, as well as by technological developments. The most influential among these were the end of the cold war and the emergence of giant, often multinational telecommunications companies or consortia, that provide services previously reserved to state monopolies. Technically, the new era can be characterized by a host of new types of satellite services, the use of digital communication techniques, the proliferation of low power devices that do not require individual licensing and by a steady move towards higher frequencies. I discuss the evolution of some of these trends and their implications for radio astronomy.
\end{abstract}

\section{Introduction}

The first attempt to detect extra-terrestrial radio emissions dates to 1890 , when Edison tried to detect radio emission from the Sun. There is no record of the results, suggesting strongly that the experiment was unsuccessful. Four years later Sir O. Lodge attempted to detect solar radio emission and documented the first instance of interference to a radio astronomy experiment. He wrote:

" I hope to try for long wave radiation from the Sun ..." "I did not succeed in this ... There were evidently too many terrestrial sources of disturbance in a city like Liverpool to make the experiment feasible." (Quoted by J. S. Hey 1973)

Radio astronomy was born with K. G. Jansky's detection of radio emission from the Galaxy (1932), while searching for the origin of weak static that was causing interference to ship-to-shore communications. At first, Jansky attributed the emission to weak, man-made interference. Finally, radio emission from the Sun was also detected, by J.S. Hey during World War II (1942), while searching for the origin of radar jamming. In Hey's words:

"In both instances, the aim has been to study types of interference

limiting the effectiveness of practical systems." (J.S. Hey 1973) 
Thus, radio astronomy has been closely linked to man- made radio interference since it's beginnings. Its future continues to depend on how effectively it can be protected by regulations or can overcome man-made radio interference by technical means.

\section{Telecommunications Trends and Forces on the Spectrum}

Table 1. The changing telecommunications environment.

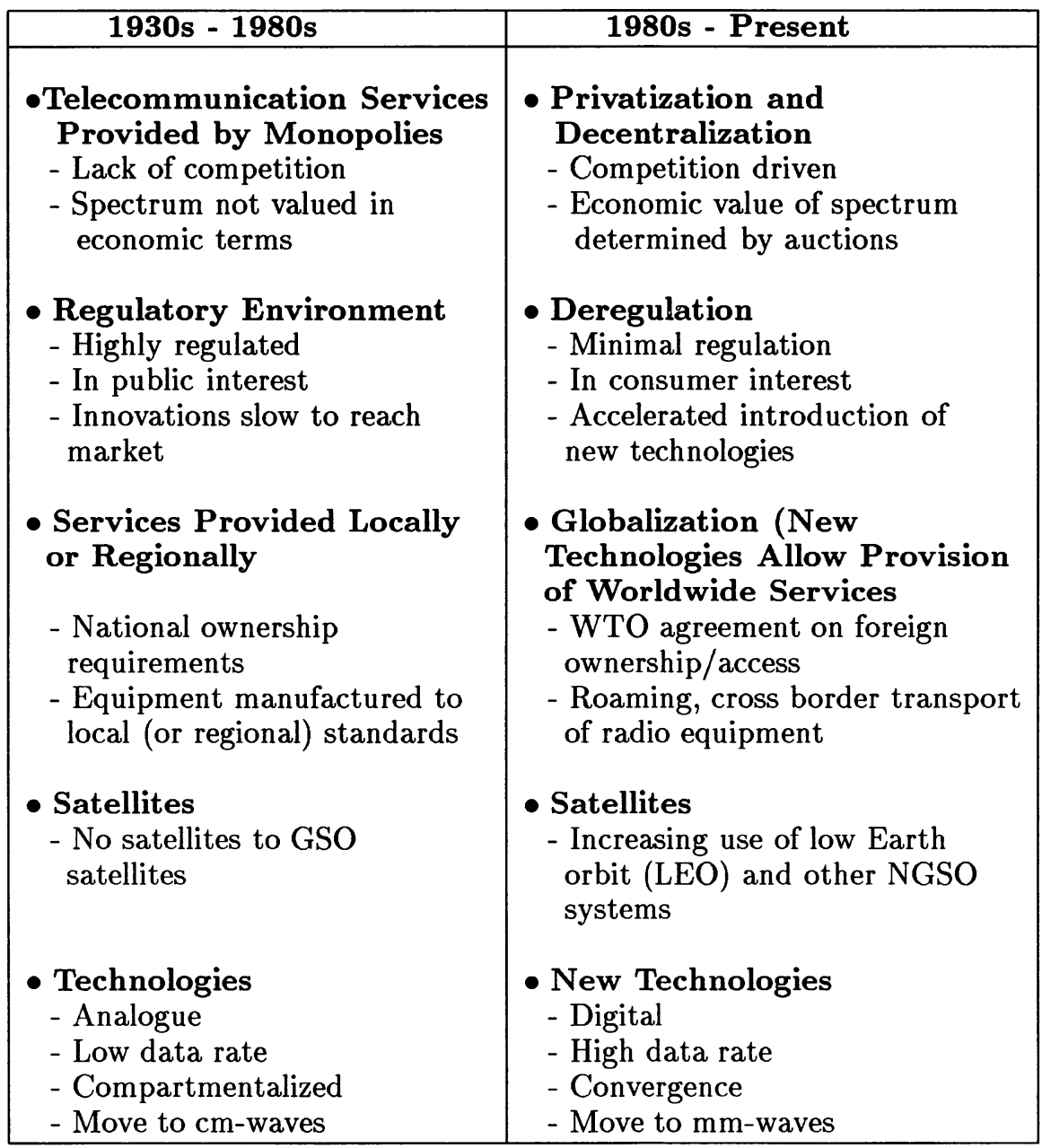

Early on, radio astronomy observatories avoided man-made interference by locating telescopes in remote areas and this strategy was mostly successful until the 1970s. During the last two decades, however, radio observatories have been 
increasingly experiencing instances of interference. In addition, satellite transmissions now restrict access to large portions of the radio spectrum, no matter how remote the observatory site.

When radio astronomy was born and matured, the telecommunications environment was dominated by state monopolies and a strong regulatory environment. Since radio astronomy observatories are, as a rule, financed and built by governments, regulatory bodies could also be relied on to provide protection from interference. Radio communications were analogue and confined to frequencies below a few hundred megahertz, a fact perpetuated in officially accepted designations, which refer to, e.g. the $3-30 \mathrm{MHz}$ and $30-300 \mathrm{MHz}$ frequency ranges as the "High Frequency" or "HF" and "Very High Frequency" or "VHF" bands, respectively. As radio technology progressed, higher frequency ranges became commercially attractive. The $1-3 \mathrm{GHz}$ range, favoured for satellite applications, became the most fought over portion of the spectrum in the 1990s. This range, of course, is also of great interest to astronomers, because it contains the HI and $\mathrm{OH}$ lines and their redshifted extensions.

Table 2. Spectrum management principles used to formulate policies of the U.S. Federal Communications Commission (FCC)

\section{- COMPETITION}

- Rely on market forces to ensure economically efficient use of spectrum

- Avoid mandating specific services

- Minimize regulations that limit competition, obstruct innovation, or impede efficient investment

\section{- FLEXIBILITY}

- Establish standards sparingly

- PUBLIC INTEREST

- Where the market is unlikely to produce essential public benefits in adequate quantities, minimum intervention may apply to ensure that these benefits are achieved

- Spectrum set asides for public services or benefits

- LICENSING AND FEE POLICIES

- Support spectrum value

- ADMINISTRATIVE CERTAINTY

- Establish firm ground rules on interference

- GLOBAL MARKET CONTEXT

- Encourage efficient worldwide spectrum use to ensure spectrum availability

- Promote seamless, worldwide networks

- Coordinate with other nations (satellites)

The global telecommunications environment begun to change in the 1960's and has undergone a revolution during the last decades. Impulse for this revolution continues to be provided by political and technological developments, e.g. the end of the cold war and the emergence of giant, often multinational 
companies or consortia, that provide services previously reserved to state monopolies. Some changing telecommunications trends that affect radio astronomy are summarized in Table 1.

In terms of technical developments, the new era can be characterized by the advent of satellite communications and the increasing use of digital modulation techniques. These changing trends affect the use of the radio spectrum. The principles that guide spectrum management and use, particularly by the private sector in the U.S.A., have been summarized by the Federal Communications Commission (FCC) as shown in Table 2. It is fair to say that to a greater or lesser degree these principles represent current trends, worldwide.

\section{The Impact on Radio Astronomy}

Clearly, radio astronomy is impacted by these telecommunications trends, particularly by: 1) the large increase in the numbers (and power) of satellite borne transmitters, 2) the failure of satellite transmitters to curb their unwanted (outof-band and spurious) emissions and 3) the increasing use of the mm-wave spectral range (say, above $30 \mathrm{GHz}$ ).

\subsection{Increase in the Number of Satellite-Borne Transmitters}

The sensitivity of radio astronomy observations can be improved by decreasing the system temperature or by increasing the receiver bandwidth or observing time. For many radio astronomy systems it is difficult to decrease further the system temperature. In some cases, at least for the receiver, theoretical limits have been reached. The sensitivity of an observation is inversely proportional to the square root of the observing time multiplied by the receiver bandwidth. For continuum observations a $10 \%$ bandwidth is desirable, but most radio astronomy allocations below $70 \mathrm{GHz}$ are much narrower, in fact, only a couple are close to $2 \%$. For this reason, radio astronomers often make use of all available spectrum and do not confine themselves to allocated radio astronomy bands. Unfortunately, proliferation of satellite transmitters, particularly in the $1-3 \mathrm{GHz}$ band makes it increasingly difficult to access bands wider than the narrow bands allocated to radio astronomy. Ultimately, sensitivity that is lost due to a decrease in bandwidth can only be made up by increasing the observing time, thus resulting in higher telescope oversubscription rates on existing telescopes.

The increase in the number of satellites over the last 25 years can be illustrated by the increase in advance published Geostationary Orbit satellite systems (GSOs) at the International Telecommunication Union (ITU), shown in Fig. 1. Many advanced published satellites never make it to the launch pad; they are used as placeholders, to keep someone else from occupying an orbital slot. Nevertheless, the exponential growth is indicative of the desire by many countries to introduce more and more satellite-based services. The problem is further compounded by the numerous Non-Geostationary (NGSO) systems now operating or planned. These systems consist of large numbers of satellites and, as a rule, are designed to provide global services, so several satellites are always visible from any point on Earth.

A somewhat more realistic way of visualizing the diminishing fraction of spectrum to which astronomers have access is shown in Figure 2, that illustrates 


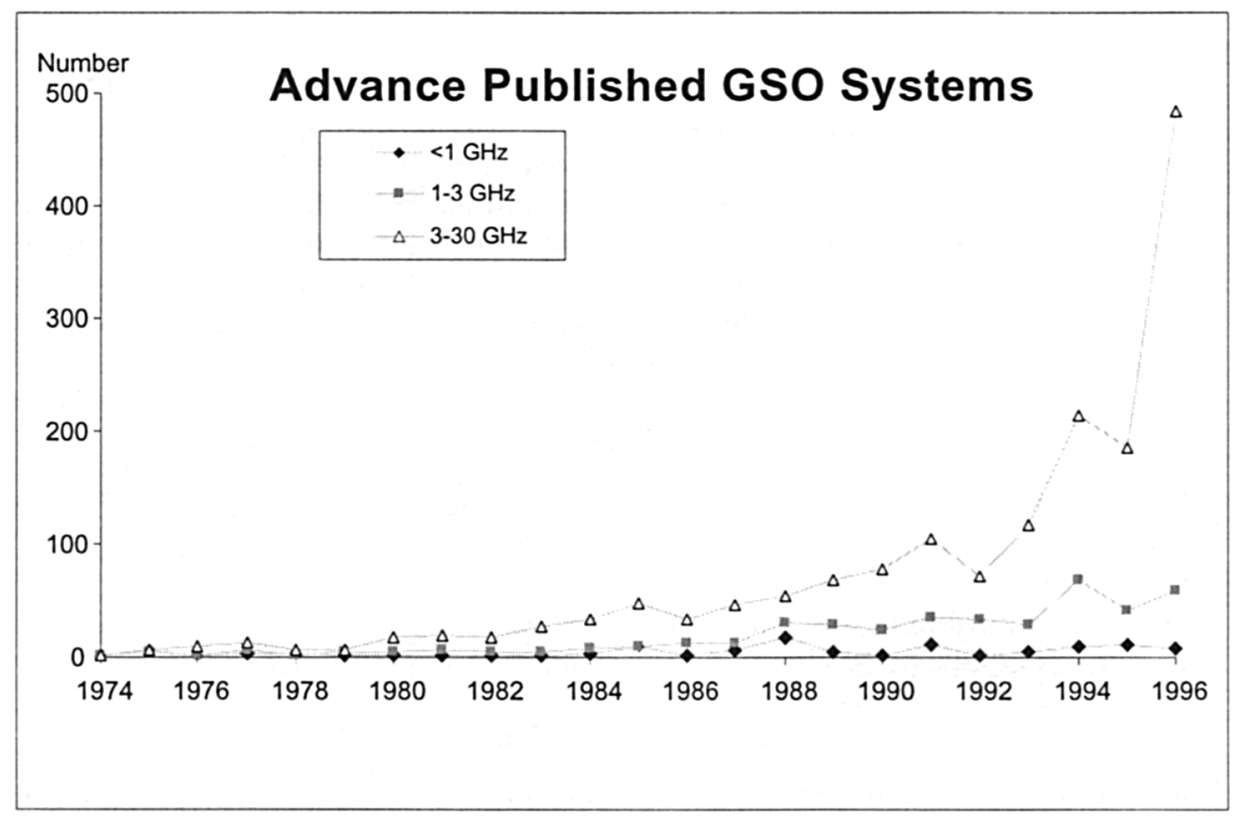

Figure 1. Advance published GSO systems at the ITU $1974-1996$.

the variation in the amount of spectrum allocated to the fixed service and to satellite downlinks over the last three decades. Sharing the spectrum between radio astronomy observatories and terrestrial transmitters, particularly those in the fixed service, is relatively easy, through geographical separation. Sometimes other means can also be used, such as placing a null in the transmitting antenna pattern in direction of the affected radio observatory. On the other hand, in-band sharing between a satellite downlink and a radio observatory is not possible, as long as the satellite transmits while above the observatory's horizon (and sometimes even when the satellite is below the horizon, due to troposcatter and other atmospheric effects). $485 \mathrm{MHz}$ of additional spectrum was allocated to satellite downlinks between 1969 and 1992, in the $1-3 \mathrm{GHz}$ range. Most of this came from spectrum that was previously allocated to the fixed service, which lost $476 \mathrm{MHz}$ over the same period. Radio astronomers can no longer plan on access to the spectrum allocated to downlinks, possibly from any place on Earth.

\subsection{Unwanted Emissions}

Clearly, it is impossible for radio astronomers to observe in frequency bands that are allocated to satellite downlinks. Unwanted emissions of satellite transmitters often spill over into adjacent and neighboring frequency bands, at levels that render them unusable for radio astronomy observations. Recent examples include the GLONASS and Iridium satellite systems. With the proliferation of satellite systems, particularly NGSOs, more problems are likely to occur. Table 3 shows 


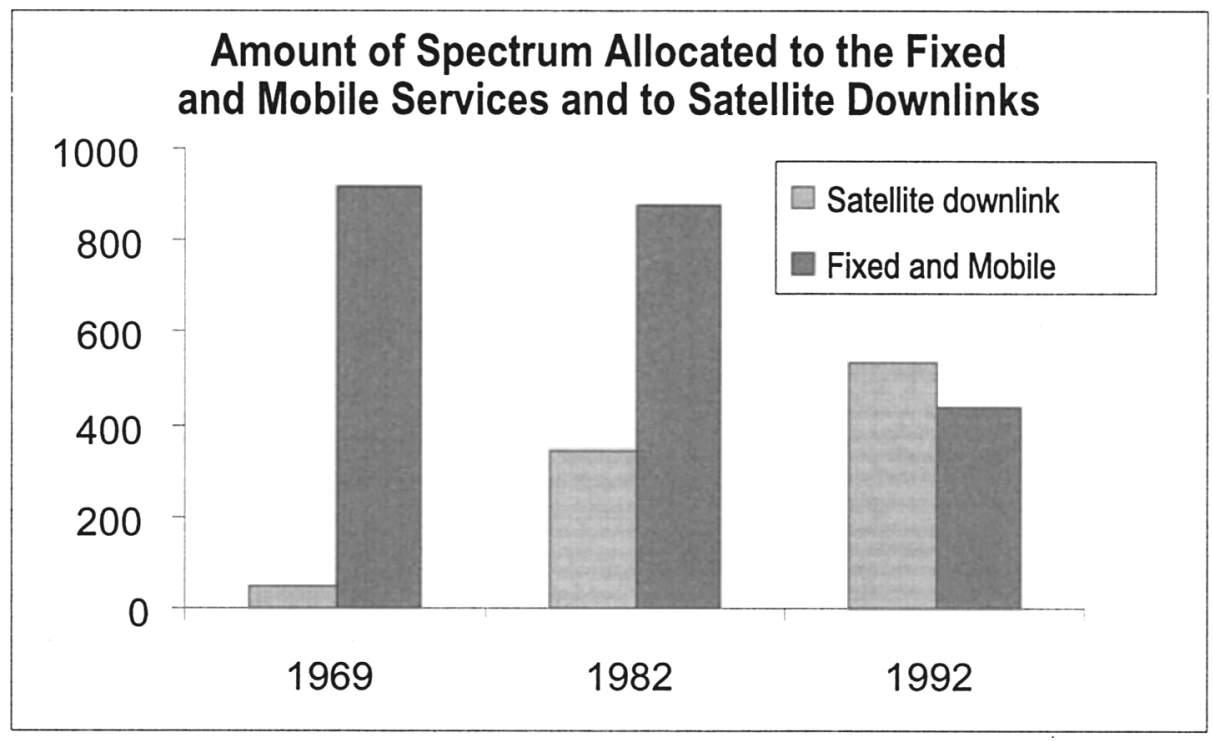

Figure 2. The changing amount of spectrum allocated to fixed and mobile services and to satellite downlinks.

existing and planned satellite service downlinks next to or near primary radio astronomy bands in the $100 \mathrm{MHz}$ to $30 \mathrm{GHz}$ range.

Proposals have also been made recently for downlink allocations next to the 406.1-410 MHz and $1400-1427 \mathrm{MHz}$ bands and although they were not adopted, there can be little doubt that such proposals will continue to be advanced. For example, WRC-97 allocated the 40.5 - $42.5 \mathrm{GHz}$ band, adjacent to the 42.5 $43.5 \mathrm{GHz}$ primary radio astronomy band, to the Fixed Satellite Service (FSS). The allocation is conditioned, however, to finding ways to share the spectrum between radio astronomers and the FSS.

Can the situation be improved? Radio astronomers sought to place limits on unwanted emissions, particularly on those originating from space-based transmitters. Until recently, there were no recommended limits at all on transmitters operating above $17.7 \mathrm{GHz}$. Spurious emissions of terrestrial transmitters are now subject to limits, but space-based transmitters are still subject only to "design objectives", that are expected to be converted into hard limits at WRC-2000. These limits are, however, far from sufficient to protect nearby radio astronomy bands. Increased use of the efficient modulation methods by satellite designers could improve the situation greatly. Martin, Yan and Lam (1997) compared available modulation methods and showed that at the $60 \mathrm{~dB}$ attenuation level, the occupied bandwidth varies between the most and the least efficient methods by a factor of 20. Out-of-band emissions are certainly of the most serious concern to radio astronomers, but use of the more efficient modulation methods would benefit all services in an era of spectrum scarcity and would result in substantial cost savings. 
Table 3. Existing and planned satellite service downlinks next to or near primary radio astronomy bands in the $100 \mathrm{MHz}$ to $30 \mathrm{GHz}$ range.

\begin{tabular}{|c|c|c|c|}
\hline $\begin{array}{c}\text { PRIMARY } \\
\text { RADIO } \\
\text { ASTRONOMY } \\
\text { BAND }\end{array}$ & $\begin{array}{c}\text { ADJACENT } \\
\text { SPACE-TO- } \\
\text { EARTH BAND }\end{array}$ & $\begin{array}{c}\text { SPACE } \\
\text { SERVICE }\end{array}$ & $\begin{array}{c}\text { CLOSEST } \\
\text { DOWNLINK }\end{array}$ \\
\hline $\begin{array}{c}150.05-153 \mathrm{MHz} \\
(\mathrm{R} 1)\end{array}$ & $149-150.05 \mathrm{MHz}$ & $\begin{array}{l}\text { Radionav } \\
\text { Sat }\end{array}$ & \\
\hline $322-328.6 \mathrm{MHz}$ & none & & $\begin{array}{c}\text { 387-390 MHz } \\
\text { mss }\end{array}$ \\
\hline $406.1-410 \mathrm{MHz}$ & none* & & $\begin{array}{c}\text { 401-402 MHz } \\
\text { Space Ops }\end{array}$ \\
\hline $608-614 \mathrm{MHz}$ & none & & $\begin{array}{c}460-470 \mathrm{MHz} \\
\text { MetSat }\end{array}$ \\
\hline $1400-1427 \mathrm{MHz}$ & none $^{*}$ & & $\begin{array}{c}1452-1492 \mathrm{MHz} \\
\text { BSS }\end{array}$ \\
\hline $1610.6-1613.8 \mathrm{MHz}$ & $1613.8-1626.5 \mathrm{MHz}$ & $\mathrm{mss}$ & \\
\hline $1660-1670 \mathrm{MHz}$ & $1670-1710 \mathrm{MHz}$ & Met Sat & \\
\hline $2690-2700 \mathrm{MHz}$ & $2670-2690 \mathrm{MHz}$ & MSS (R 2) & \\
\hline $4990-5000 \mathrm{MHz}$ & none* & & $\begin{array}{c}4500-4800 \mathrm{MHz} \\
\text { FSS } \\
\end{array}$ \\
\hline $10.68-10.7 \mathrm{GHz}$ & $10.7-11.7 \mathrm{GHz}$ & FSS & \\
\hline $15.35-15.4 \mathrm{GHz}$ & $15.4-15.7 \mathrm{GHz}$ & FSS & \\
\hline $22.21-22.5 \mathrm{GHz}$ & none & & $\begin{array}{c}21.4-22.0 \mathrm{GHz} \\
\text { BSS (R1 and R3) }\end{array}$ \\
\hline $23.6-24 \mathrm{GHz}$ & $24-24.05 \mathrm{GHz}$ & $\begin{array}{l}\text { Amateur } \\
\text { Sat }\end{array}$ & \\
\hline
\end{tabular}

${ }^{*}$ Downlink recently proposed in nearby band.

\subsection{Increasing Use of the Mm-Wave Spectral Range}

Most of the mm-wave spectrum is of interest to astronomers. Over 2,100 spectral lines of 80 chemical compounds have been identified in the $71-275 \mathrm{GHz}$ range and more have been predicted. Many of these lines are listed as being astrophysically important (Rec. ITU-R RA.314-8), as they may yield unique information on various aspects of the Universe. Astronomical observations in the mm-region require as wide receiver bandwidths as possible, to enable continuum observations of faint objects, such as extrasolar planets or very distant galaxies. Very sensitive observations are possible, using bolometer detectors with bandwidths of several tens of $\mathrm{GHz}$ and such devices are in use at several observatories. Wide frequency coverage is also needed to observe highly red-shifted objects that provide crucial information on the formation of galaxies and about the early history of the Universe, that may not be obtained at other frequencies. 
Mm-telescopes have been in operation for nearly three decades in the Americas, Asia and Europe. Reflecting the growing interest in mm-wave astronomy, the Atacama Large Millimeter Array (ALMA), a giant international project by astronomers from the U.S.A., Europe and possibly other countries that may join the collaboration, is currently under development. Several other large mm-wave telescopes and interferometers are also in the design or construction phases and are expected to begin operations in the next decade.

The spectrum above the $60 \mathrm{GHz}$ oxygen absorption bands is allocated to various radio services, including satellites, but has been scarcely used until now, except by radio astronomers and by the remote sensing community. Spectrum crowding at lower frequencies and recent technological advances have made this range more attractive for commercial use and active radio services are moving towards the millimetre waves. Protection of this spectrum for astronomical observations is therefore timely. Since there are few users yet, reaccommodation of the spectrum allocations is expected to touch fewer entrenched interests than at lower frequencies and would not require costly relocation of existing services. Because of relatively high absorption at ground level at these frequencies, it is estimated that coordination radii of the order of $100-150 \mathrm{~km}$ are required to protect observatories from terrestrial interference. Assuming, e.g., an absorption coefficient of $0.5 \mathrm{~dB} / \mathrm{km}$, spreading and propagation losses over a distance of $100 \mathrm{~km}$ amount to $150 \mathrm{~dB}$. The power flux density due to a $1 \mathrm{~kW}$ omnidirectional transmitter at a distance of $100 \mathrm{~km}$ is then typically $-120 \mathrm{~dB}\left(\mathrm{~W} / \mathrm{m}^{2}\right)$. Detrimental power flux densities for continuum radio observations in astronomy bands above $71 \mathrm{GHz}$ (listed in Rec. ITU-R RA.769-1, Table 1) range from -125 $\mathrm{dB}\left(\mathrm{W} / \mathrm{m}^{2}\right)$ at $89 \mathrm{GHz}$, to $-113 \mathrm{~dB}\left(\mathrm{~W} / \mathrm{m}^{2}\right)$ at $270 \mathrm{GHz}$. For spectral line observations values range from $-144 \mathrm{~dB}\left(\mathrm{~W} / \mathrm{m}^{2}\right)$ at $88.6 \mathrm{GHz}$ to $-131 \mathrm{~dB}\left(\mathrm{~W} / \mathrm{m}^{2}\right)$ at $265 \mathrm{GHz}$ (Table 2 of the same Recommendation). The relatively small area of the coordination regions required, coupled with the limited number of existing and planned mm-observatories and their remote locations, should have only a minimal impact on terrestrial services.

Table 4. Radio Astronomy access to mm-wave spectrum before and (hopefully) after WRC-2000

\begin{tabular}{|c|c|c|c|c|c|}
\hline Window & $\begin{array}{c}\text { Desired } \\
\text { ALMA } \\
\text { Coverage } \\
(\mathrm{GHz})\end{array}$ & $\begin{array}{c}\text { Current Radio } \\
\text { Astronomy } \\
\text { Bands } \\
(\mathrm{GHz})\end{array}$ & $\%$ & $\begin{array}{c}\text { Desired Radio } \\
\text { Astronomy } \\
\text { Bands } \\
(\mathrm{GHz})\end{array}$ & $\%$ \\
\hline $3 \mathrm{~mm}$ & $67-116$ & $\begin{array}{c}86-92 \\
105-116\end{array}$ & 35 & $76-116$ & 82 \\
\hline $2 \mathrm{~mm}$ & $125-211$ & $\begin{array}{l}164-168 \\
182-185\end{array}$ & 8 & $\begin{array}{c}123-158.5 \\
164-167 \\
200-211\end{array}$ & 57.5 \\
\hline $1 \mathrm{~mm}$ & $211-275$ & $\begin{array}{l}217-231 \\
250-252 \\
265-275\end{array}$ & 41 & $\begin{array}{c}211-231.5 \\
235-238 \\
241-275\end{array}$ & 88 \\
\hline
\end{tabular}


Line-of-sight sharing between the radio astronomy and satellite downlinks is, as a rule, not possible. Sharing may be possible, however, with some satellite systems and services consisting of a very limited number of satellites, such as Amateur Satellites or the Cloud Profiling Radar, that expect to operate above $71 \mathrm{GHz}$, eventually. The orbital elements of these systems will be known and their knowledge should permit time sharing in these bands, as radio observatories may schedule observations at times when no satellite is over the horizon, or shut down for the limited periods of time. To protect radio observatories from unwanted emissions of satellites, downlinks could be moved to near the edges of the transparent atmospheric windows, leaving their middle portion available to be shared by terrestrial services and wide band passive receivers.

Proposals to protect radio astronomy at $\mathrm{mm}$-wavelengths along these lines are currently being prepared by spectrum managers of various countries, including the U.S.A., Europe, Japan and others, to be put forward at WRC-2000. Table 4 shows the fraction of $\mathrm{mm}$-spectrum accessible to radio astronomers from a regulatory point of view now and the fraction that is expected to become accessible, if these proposals are adopted by WRC- 2000 .

\section{Summary}

Since its beginnings, radio astronomy had to develop and prosper in a noisy environment. The situation worsened considerably with the advent and proliferation of satellite systems, particularly NGSOs, which may render astronomical observations impossible to carry out in large portions of the spectrum. Technical and regulatory solutions should be sought to radio astronomy's interference problems and to preserve mankind's access to this resource. Attempts towards technical solutions are discussed elsewhere in this volume. Regulatory solutions are manpower intensive and costly. Nevertheless, if radio astronomers wish to preserve access to the spectrum, they will need to dedicate a larger share of human resources and their budget to spectrum management activities, so they can compete with the numerous other activities that claim use of the radio spectrum. Finally, education efforts will have to be undertaken or intensified, so that policy makers and the general public appreciate the value of radio astronomy and what it would mean for our understanding of the Universe to lose access to most or all of the radio spectrum. Only by pursuing all these routes are astronomers likely to retain access to crucial segments of the radio spectrum that they need.

\section{References}

Hey, J. S. 1973, The Evolution of Radio Astronomy, New York: Science History Pub.

Martin, W.L., Yan, T. and Lam, L.V. 1997, Efficient Modulation Methods Study at NASA/JPL, Space Frequency Coordination Group Doc. SF17-28/D

ITU-R Recommendations 1997, RA Series, Geneva 